\title{
Medical Costs Associated with Severity of Chronic Kidney Disease in Type 2 Diabetes Mellitus in Singapore
}

Gwyneth J Lim, ${ }^{1}$ Yan Lun Liu, ${ }^{2} M B C h B, M R C P, M P H$, Serena Low, ${ }^{3} M B B S, M S c$ (Public Health), Keven Ang, ${ }^{3}$ PhD, Subramaniam Tavintharan, ${ }^{4}{ }_{M B B S}, M R C P, M C I$, Chee Fang $\underline{\text { Sum }},{ }_{M B B S, M R C P}$, Su Chi Lim, ${ }^{3,4,5}{ }_{M B B S, M R C P, P h D}$

\begin{abstract}
Introduction: This was a retrospective cross-sectional study to assess the impact of chronic kidney disease (CKD) and its severity in Type 2 diabetes mellitus (T2DM) on direct medical costs, and the effects of economic burden on CKD related complications in T2DM in Singapore.

Methods: A total of 1,275 T2DM patients were recruited by the diabetes centre at Khoo Teck Puat Hospital from 2011-2014. CKD stages were classified based on improving global outcome (KDIGO) categories, namely the estimated glomerular filtration rate (eGFR) and albuminuria kidney disease. Medical costs were extracted from the hospital administrative database.

Results: CKD occurred in $57.3 \%$ of patients. The total mean cost ratio for CKD relative to non-CKD was $2.2(P<0.001)$. Mean (median) baseline annual unadjusted costs were significantly higher with increasing CKD severity-S\$1,523 (S\$949), S\$2,065 (S\$1,198), $\mathrm{S} \$ 3,502$ (S\$1,613), and $\mathrm{S} \$ 5,328(\mathrm{~S} \$ 2,556)$ for low, moderate, high, and very high risk respectively $(P<0.001)$. CKD $(P<0.001)$, age at study entry $(P=0.001)$, Malay ethnicity ( $P=0.035)$, duration of diabetes mellitus (DM; $P<0.001)$, use of statins/fibrates $(P=0.021)$, and modified Diabetes Complications Severity Index (DCSI) $(P<0.001)$ were positively associated with mean annual direct medical costs in the univariate analysis. In the fully adjusted model, association with mean annual total costs persisted for CKD, CKD severity and modified DCSI.

Conclusion: The presence and increased severity of CKD is significantly associated with higher direct medical costs in T2DM patients. Actively preventing the occurrence and progression in DM-induced CKD may significantly reduce healthcare resource consumption and healthcare costs.
\end{abstract}

Ann Acad Med Singap 2020;49:731-41

Keywords: Chronic kidney disease, costs, endocrinology, nephrology

\section{Introduction}

The global prevalence of diabetes mellitus (DM) is projected to increase from 451 million in 2017 to 693 million in 2045, with the prevalence of Type 2 DM (T2DM) in Singapore doubling from $7.3 \%$ in 1990 to $15 \%$ in $2050 .^{1,2} \mathrm{~A}$ recent study revealed that the total economic cost of DM to Singapore constituted about $10 \%$ of total healthcare expenditure in $2010 .^{3}$ The cost is projected to increase from US\$787 million in 2010 to US $\$ 1,867$ million in $2050 .^{3}$

It has been established that diabetic complications substantially heightened the economic costs of T2DM. ${ }^{4-6}$ A European study discovered that up to $40 \%$ of T2DM patients are suffering from Chronic Kidney Disease (CKD). ${ }^{7}$ In 2015, Singapore reported that $66 \%$ of patients with newly diagnosed end-stage renal disease

\footnotetext{
${ }^{1}$ Lee Kong Chian School of Medicine, Nanyang Technological University

${ }^{2}$ Department of Medicine, Khoo Teck Puat Hospital

${ }^{3}$ Clinical Research Unit, Khoo Teck Puat Hospital

${ }^{4}$ Diabetes Centre, Admiralty Medical Centre

${ }^{5}$ Saw Swee Hock School of Public Health, National University of Singapore

Address for Correspondence: A/ Prof Su Chi Lim, Diabetes Centre, Admiralty Medical Centre, 676 Woodlands Drive 71, \#03-01 Kampung Admiralty, Singapore 730676.

Email address: lim.su.chi@ktph.com.sg
} 
(ESRD) were secondary to DM, among one of the highest proportions globally. ${ }^{8}$ It is evident that the economic burden from CKD and ESRD in DM is remarkably heavy due to its high prevalence and complexities of disease management. ${ }^{69}$ We earlier reported that medical costs increased proportionately with CKD progression. ${ }^{10}$

There have been multiple studies that examined the direct costs of CKD in T2DM, wherein the definitions of CKD were based on estimated glomerular filtration rates (eGFR) alone, levels of proteinuria alone, selfreport, or population attributable risk..$^{9,11-16}$ Studies linked with medical costs and CKD severity in DM patients according to definitions and classifications from Kidney Disease: Improving Global Outcomes (KDIGO) Clinical Practice Guideline for Evaluation and Management of $\mathrm{CKD}^{17}$ (diagnosis of CKD by a matrix of eGFR and albuminuria measurements) remain relatively scarce. ${ }^{18}$ Jointly assessing renal function based on eGFR and albuminuria provides a more accurate reflection on health resource consumption, where for instance, the subgroup of individuals with substantial albuminuria but preserved eGFR may consume significantly more health resources due to their cardiovascular disease burden. In addition, limited data is available to demonstrate the effects of various DM complications in economic terms, which can facilitate objective assessment of healthcare resource utilisation, particularly for patients with CKD. One of the commonly used tools in this area is the Diabetes Complication Severity Index (DCSI). ${ }^{19,20}$

To the best of our knowledge, there is no published study on medical cost and severity of CKD according to both eGFR and albuminuria in T2DM. Hence, this study aims to assess the impact of CKD severity in T2DM on direct medical costs based on KDIGO guidelines in Singapore, a multi-ethnic society where diabetic ketoacidosis (DKD) prevalence is high. This study also evaluates the economic burden on CKD-related complications in T2DM by DCSI. The findings will serve as baseline reference for future cost-of-illness studies, especially pre-2011 and post-introduction of sodium-glucose co-transporter-2 (SGLT2) inhibitor, as well as future economic evaluation on intervention to prevent DM complications in Singapore.

\section{Methods}

This was a retrospective cross-sectional study on patients with T2DM attending a diabetes centre in Khoo Teck Puat Hospital. These patients were from the Singapore Study of Macro-angiopathy and Micro- vascular Reactivity in Type 2 Diabetes (SMART2D), a cross-sectional study of adults aged 21-90 years with T2DM that was conducted between August 2011 and February 2014..$^{21}$ The exclusion criteria were as follows: T1DM, pregnancy, active inflammation, cancer, on non-steroid anti-inflammatory drugs (NSAIDS) on the day of the assessment, on oral steroids equivalent to $>5 \mathrm{mg} /$ day of prednisolone, fasting glucose $<4.5 \mathrm{mmol}$ or $>15.0 \mathrm{mmol}$, HbAlc $>12 \%$, inability to give informed consent, and insertion of pacemaker or any device that may be affected by electric current. Ethics approval was obtained from the National Healthcare Group Domain Specific Review Board and the study was conducted in accordance with the Declaration of Helsinki. Written informed consent was obtained from all subjects prior to enrolment in the study. There were a total of 1,275 patients with cost data available. Demographical and clinical data were obtained by trained nurses from patients' case records and a standard questionnaire administered to the patients.

CKD was defined as abnormalities of kidney structure, namely, one or more of the following: albuminuria (albumin to creatinine ratio $\geq 30 \mathrm{mg} / \mathrm{g}$ ), urine sediment abnormalities, electrolyte and other abnormalities due to tubular disorders, abnormalities detected by histology, structural abnormalities detected by imaging, history of kidney transplantation, or kidney function issue (decreased eGFR $<60 \mathrm{~mL} / \mathrm{min} / 1.73 \mathrm{~m}^{2}$ ) present for $>3$ months, with implications for health. CKD was classified based on eGFR (G1: $\geq 90 \mathrm{~mL} / \mathrm{min}$ per $1.73 \mathrm{~m}^{2}$; G2: $60-89 \mathrm{~mL} / \mathrm{min}$ per $1.73 \mathrm{~m}^{2}$; G3a: $45-59 \mathrm{~mL} / \mathrm{min}$ per $1.73 \mathrm{~m}^{2}$; G3b: $30-44 \mathrm{~mL} / \mathrm{min}$ per $1.73 \mathrm{~m}^{2}$; G4: $15-29 \mathrm{~mL} /$ min per $1.73 \mathrm{~m}^{2}$; Stage $\mathrm{G} 5:<15 \mathrm{~mL} / \mathrm{min}$ per $1.73 \mathrm{~m}^{2}$ ) and albuminuria (A1: $<30 \mathrm{mg} / \mathrm{g} ; \mathrm{A} 2: 30-300 \mathrm{mg} / \mathrm{g} ; \mathrm{A} 3$ : $>300 \mathrm{mg} / \mathrm{g}$ ) categories, as stipulated in the KDIGO Clinical Practice guideline. The outcome was the severity of CKD, of which eGFR and albuminuria categories with similar relative risk for CKD outcomes were grouped into risk categories-low risk, moderately increased risk, high risk, and very high risk.

Neuropathy was assessed with a neurothesiometer (Horwell Scientific, Yorkshire, UK) for vibration and with a $10 \mathrm{~g}$ monofilament for light touch. Neuropathy is present if an abnormal finding in monofilament (inability to detect at least 2 of 10 points on either foot) or neurothesiometer testing of $\geq 25$ volts on either foot was detected. Foot examination was performed by the same team of research nurses who received standardised training and accreditation. Peripheral arterial disease (PAD) was assessed as follows: Ankle Brachial Index 
(ABI) was calculated as the ratio of the higher of the two systolic pressures (from posterior tibial and dorsalis pedis) at the ankle to the higher of the right and left brachial artery pressures, as previously reported. ${ }^{22}$ PAD is defined to be present if the lower $\mathrm{ABI} \leq 0.9$ or if patients had previous amputations. ${ }^{23}$ The patients were additionally classified with borderline abnormal $\mathrm{ABI}$ as $0.91 \leq \mathrm{ABI} \leq 0.99$ using the latest $\mathrm{ACCF} / \mathrm{AHA}$ guidelines. ${ }^{24}$ Patients with $\mathrm{ABI}>1.4$ were excluded from analyses.

Modified DCSI was derived from clinical measurements, laboratory data and International Classification of Diseases Tenth Revision, Australian Modification (ICD-10-AM) with reference on the classification from previous studies. ${ }^{19,25,26}$ For the purpose of this analysis where CKD risk was the exposure of interest, we did not include nephropathy in the score. The modified DCSI comprises 6 categories of complications and their severity levels: retinopathy, neuropathy, cerebrovascular, cardiovascular, peripheral vascular disease and metabolic condition. Each complication was categorised into 2 or 3 levels (normal $=0$, mild $=1$, severe $=2$ ). We also used $\mathrm{HbAlc}$ (HbAlc level $\leq 7.0 \%, 7.1-9.0 \%$ and $>9.0 \%$ ) instead of metabolic events (ketoacidosis, hyperosmolar and other coma) for metabolic component of the modified DCSI score as the HbAlc level would reflect the metabolic control. Information on cardiovascular disease and stroke were obtained from self-report in the questionnaire and extracted from International Classification of Diseases Tenth Revision, Australian Modification (ICD-10-AM).

A prevalence-based epidemiological approach adopting a bottom-up methodology was used to estimate direct medical costs. Costs were extracted from administrative database for inpatient, outpatient, day surgeries and Accident and Emergency (A\&E) visits from 2011 to 2014. These included physician visits, investigations, allied health services, nurse education, medications, consumables and procedures. Direct non-medical costs (i.e. transport expenses), and indirect costs (i.e. lost productivity, quality of life) were not included.

Direct medical costs were measured by using the total charges before subsidy, which is the total medical bill before any deduction from government subsidies or insurance claims. All costs were expressed in year 2014 Singapore dollars (SGD). Consumer price index was used to estimate values older than 2014.

Categorical data were expressed as a percentage and continuous data as means \pm standard deviation
(SD) unless otherwise stated. Differences in patient characteristics, risk factors, medications, complications and healthcare utilisation among categories of risk for CKD outcomes (low, moderate, high, and/very high risk) were studied using chi-square test for categorical variables, and one-way ANOVA or Kruskal Wallis for continuous variables where appropriate.

Generalised linear models with Gaussian distribution and log-link function were used to examine the relationship between CKD, CKD severity and annual direct medical costs, adjusting for covariates with $P$-value $<0.1$ in the univariate analysis. The covariates include age, ethnicity, duration of DM, use of renin-angiotensin system (RAS) antagonist, DM treatment, use of statins/fibrates and log-transformed modified DCSI score.

All statistical analyses were performed using STATA version 14.0 (StataCorp, College Station, US). A two-tailed $P$-value $<0.05$ was considered statistically significant.

\section{Results}

\section{Patient characteristics}

Out of the 1,275 T2DM patients included in this study, CKD occurred in $57.3 \%$ of them. The distribution of CKD severity was as follows: low, $42.7 \%$; moderate, $25.9 \%$; high, $14.8 \%$; and very high, $16.7 \%$. The baseline characteristics are shown in Tables 1 and 2. Patients with more severe CKD were older; had longer duration of DM, more adverse metabolic profile in terms of body mass index (BMI), systolic blood pressure (SBP) and HbAlc; and a higher modified DCSI score $(P<0.001)$. The percentage of patients prescribed RAS antagonist, oral DM medication together with insulin, and lipid lowering agents, increased with increasing severity of CKD risk categories $(P<0.001)$.

The CKD group had more outpatient visits and inpatient hospitalisations per year compared to the non-CKD groups $(P<0.05)$. Patients with higher risk CKD also utilised more healthcare resources in terms of outpatient visits, hospitalisations, emergency visits and length of stay $(P<0.001)$ (Table 3$)$. Increasing mean length of stay, inpatient, and outpatient episodes were also observed across increasing risk of CKD.

\section{Medical cost - non-CKD versus $C K D$}

The mean annual costs for non-CKD and CKD were $\mathrm{S} \$ 1,523(95 \%$ CI $\mathrm{S} \$ 1,340-1,704)$ and $\mathrm{S} \$ 3,385(95 \%$ CI S\$2,972-3,799), respectively. The cost for CKD was $\$ 1,862$ (2.2 times) higher than the cost for non-CKD 
Table 1. Patient characteristics by chronic kidney disease $(n=1275)$

\begin{tabular}{|c|c|c|c|c|}
\hline Variables & All & No & Yes & $P$-value \\
\hline Number & 1275 & 544 & 731 & \\
\hline Entry age (years) & $56.0 \pm 11.5$ & $53.5 \pm 11.6$ & $57.9 \pm 11.0$ & $<0.001$ \\
\hline Male (\%) & $722(56.6)$ & $312(57.4)$ & $410(56.1)$ & 0.652 \\
\hline Ethnicity (\%) & & & & $<0.001$ \\
\hline Chinese & $651(51.1)$ & $294(54.0)$ & $357(48.8)$ & \\
\hline Malay & $289(22.7)$ & 75 (13.8) & $214(29.3)$ & \\
\hline Indian & $287(22.5)$ & $149(27.4)$ & $138(18.9)$ & \\
\hline Other & $48(3.8)$ & $26(4.8)$ & $22(3.0)$ & \\
\hline Duration of DM (years) & $12.2 \pm 9.4$ & $9.7 \pm 8.5$ & $14.1 \pm 9.7$ & $<0.001$ \\
\hline SBP (mmHg) & $141.9 \pm 19.7$ & $134.0 \pm 15.5$ & $147.9 \pm 20.5$ & $<0.001$ \\
\hline HbA1c (\%) & $8.0 \pm 1.4$ & $7.8 \pm 1.4$ & $8.1 \pm 1.4$ & $<0.001$ \\
\hline LDL-C (mmol/l) & $2.8 \pm 0.9$ & $2.8 \pm 0.8$ & $2.8 \pm 0.9$ & 0.445 \\
\hline $\operatorname{eGFR}\left(\mathrm{ml} / \mathrm{min} / 1.73 \mathrm{~m}^{2}\right)$ & $91.2(64.0-105.0)$ & $100.1(88.7-109.1)$ & $73.0(47.3-99.1)$ & $<0.001$ \\
\hline Urinary ACR (mg/g) & $35(10-238)$ & $9(4-16)$ & $165(54-693)$ & $<0.001$ \\
\hline Use of RAS (\%) & $824(64.8)$ & $245(45.3)$ & $579(79.3)$ & $<0.001$ \\
\hline DM Treatment (\%) & & & & $<0.001$ \\
\hline No meds & $56(4.4)$ & $33(6.1)$ & $23(3.2)$ & \\
\hline Oral only & $734(57.8)$ & $364(67.3)$ & $370(50.8)$ & \\
\hline Use of Statins/Fibrates Medications (\%) & $1065(83.7)$ & $417(76.8)$ & $648(1065)$ & $<0.001$ \\
\hline Modified DCSI & $2(1-3)$ & $1(0-2)$ & $2(1-3)$ & $<0.001$ \\
\hline
\end{tabular}

ACR: albumin-to-creatinine ratio; BMI: body mass index; DCSI: diabetes complications severity index; DM: diabetes mellitus; eGFR: estimated glomerular filtration rate; HbA1c: haemoglobin A1c; LDL-C: low density lipoprotein cholesterol; RAS: renin-angiotensin system antagonist; SBP: systolic blood pressure

(95\% CI $\$ 1,360-2,367)(P<0.001)$. For the non-CKD group, outpatient costs were highest, followed by inpatient costs, and emergency costs. As for the CKD group, inpatient costs were highest, followed by outpatient costs and emergency costs. The cost breakdown for outpatient components was similar for both non-CKD and CKD groups: medications cost most ( $38 \%$ vs $39 \%$ ), followed by investigations ( $28 \%$ vs $29 \%$ ), doctor visits $(17 \%$ vs $16 \%)$ and finally allied health visits $(11 \%$ vs $11 \%)$.

\section{Medical costs by CKD risk categories}

The mean (median) [95\% CI] baseline unadjusted costs per annum were significantly higher with increasing severity of CKD-\$1,523 (\$949) [95\% CI $\$ 1,340-1,704]$, $\$ 2,065(\$ 1,198)$ [95\% CI $\$ 1,724-$ $2,406], \$ 3,502(\$ 1,613)$ [95\% CI $\$ 2,649-4,356]$, and $\$ 5,328(\$ 2,556)[95 \%$ CI $\$ 4,295-6,361]$ for low, moderate, high, and very high risk respectively $(P<0.001)$. Similar trends of increase were observed for inpatient, outpatient, and A\&E costs (Table 4). Compared to low-risk CKD, the mean annual costs for moderate-risk, high-risk and very high-risk CKD were $\$ 543$ (1.5 times; 95\% CI $\$ 189-896 ; P=0.003$ ), $\$ 1,980$ (2.3 times; 95\% CI $\$ 1,393-2,567 ; P<0.001)$ and $\$ 3,806$ (3.5 times; $95 \% \mathrm{CI} \$ 3,100-4,511 ; P<0.001$ ) higher.

In terms of inpatient care, the mean annual costs for moderate, high-risk and very high-risk CKD were $\$ 386$ 


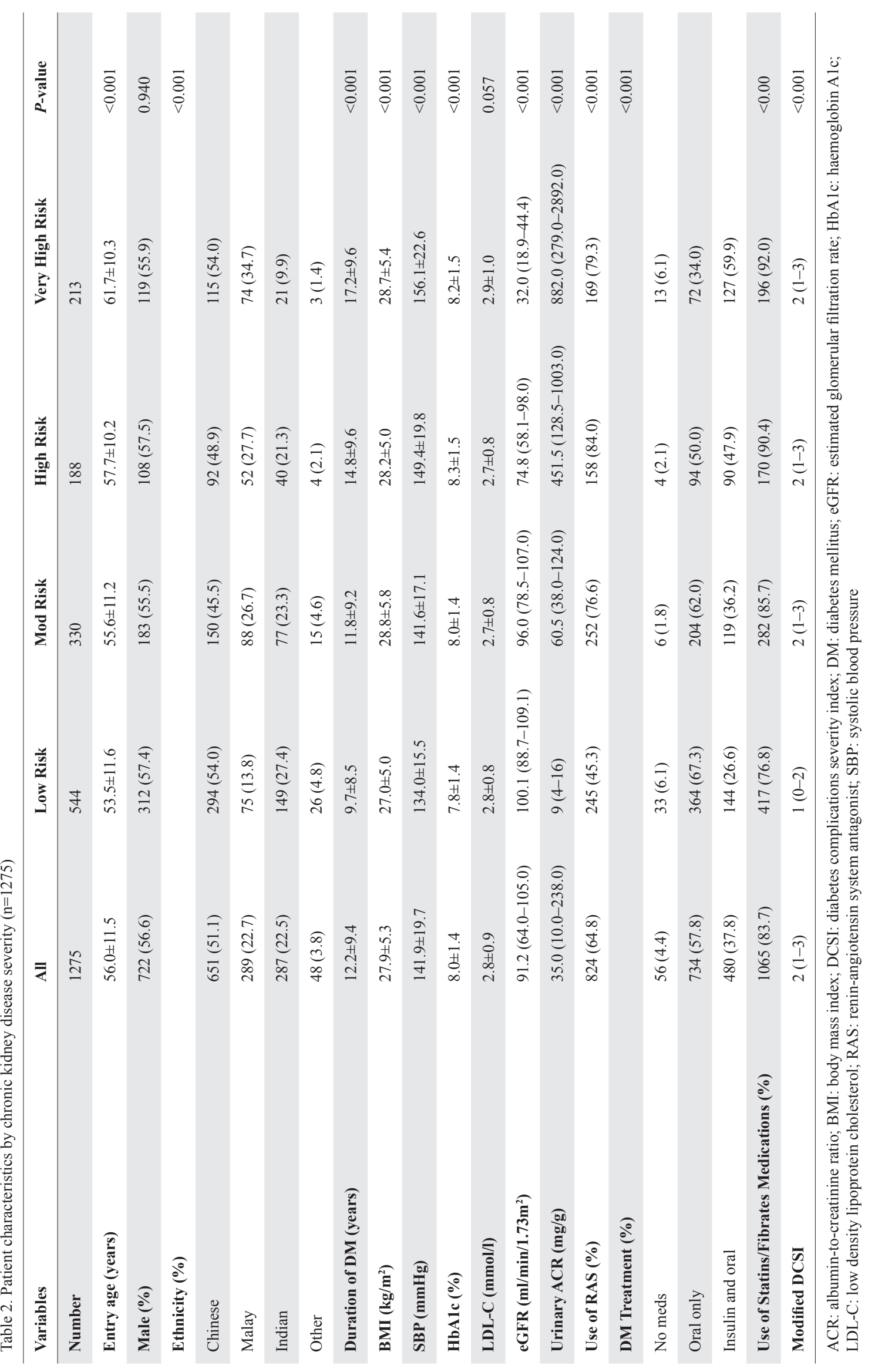


Table 3. Healthcare utilisation by CKD and CKD risk categories $(\mathrm{n}=1275)$

\begin{tabular}{|c|c|c|c|c|c|}
\hline & \multicolumn{2}{|c|}{ CKD } & & & \multirow[b]{2}{*}{$P$-value } \\
\hline & No & Yes & & & \\
\hline Number of outpatient visits & $6.6 \pm 6.3$ & $9.4 \pm 7.4$ & & & $<0.001$ \\
\hline Number of A\&E visits & $1.7 \pm 1.7$ & $2.0 \pm 2.0$ & & & 0.073 \\
\hline Number of hospitalisations & $1.2 \pm 0.5$ & $1.7 \pm 1.2$ & & & 0.004 \\
\hline \multirow[t]{2}{*}{ Length of stay (days) } & $2.1 \pm 3.2$ & $5.5 \pm 10.8$ & & & 0.022 \\
\hline & Low Risk & Mod Risk & High Risk & Very High Risk & \\
\hline Number of outpatient visits & $6.6 \pm 6.3$ & $7.5 \pm 5.8$ & $9.5 \pm 8.1$ & $12.2 \pm 8.0$ & $<0.001$ \\
\hline Number of A\&E visits & $1.7 \pm 1.7$ & $1.5 \pm 0.8$ & $1.9 \pm 1.4$ & $2.8 \pm 2.9$ & $<0.001$ \\
\hline Number of hospitalisations & $1.2 \pm 0.5$ & $1.4 \pm 0.7$ & $1.5 \pm 1.0$ & $2.1 \pm 1.4$ & $<0.001$ \\
\hline
\end{tabular}

A\&E: Accident and Emergency; CKD: chronic kidney disease

Table 4. Cost in SGD stratified by CKD severity $(n=1275)$

\begin{tabular}{|c|c|c|c|c|}
\hline & Low Risk & Mod Risk & High Risk & Very High Risk \\
\hline $\mathrm{n}$ & 544 & 330 & 188 & 213 \\
\hline \multicolumn{5}{|l|}{ Cost variables } \\
\hline \multicolumn{5}{|l|}{ Overall } \\
\hline Mean & 1523 & 2065 & 3502 & 5328 \\
\hline Median & 949 & 1198 & 1613 & 2556 \\
\hline Interquartile range & $461-1669$ & $652-2138$ & $984-3531$ & $1501-5242$ \\
\hline $90 \%$ percentile & 3037 & 4201 & 7108 & 15273 \\
\hline \multicolumn{5}{|l|}{ Inpatient } \\
\hline Standard Deviation & 1856 & 2734 & 4903 & 6893 \\
\hline Median & 0 & 0 & 0 & 0 \\
\hline $90 \%$ percentile & 0 & 2941 & 4858 & 10134 \\
\hline \multicolumn{5}{|l|}{ Outpatient } \\
\hline Mean & 972 & 1115 & 1573 & 2094 \\
\hline Standard Deviation & 802 & 792 & 1438 & 1287 \\
\hline Median & 816 & 975 & 1272 & 1818 \\
\hline $90 \%$ percentile & 1975 & 2151 & 2820 & 3697 \\
\hline \multicolumn{5}{|l|}{$\mathrm{A} \& \mathrm{E}$} \\
\hline Median & 0 & 0 & 0 & 0 \\
\hline $90 \%$ percentile & 423 & 462 & 738 & 961 \\
\hline
\end{tabular}

A\&E: Accident and Emergency; CKD: chronic kidney disease 
(1.9 times; 95\% CI $\$ 81-691 ; P=0.013$ ), $\$ 1,249$ (3.9 times; $95 \%$ CI $\$ 758-1,739 ; P<0.001)$ and $\$ 2,441$ (6.6 times; $95 \%$ CI $\$ 1,810-3,072 ; P<0.001)$ higher than that for low-risk CKD. The increase in costs were relatively smaller for outpatient and emergency visits. Outpatient mean annual costs for moderate-risk, high-risk and very high-risk CKD were $\$ 143$ (1.2 times; 95\% CI \$34-252; $P=0.010$ ), $\$ 601$ (1.6 times; 95\% CI \$434-767; $P<0.001)$ and $\$ 1,122(2.2$ times; $95 \%$ CI $\$ 966-1,275 ; P<0.001)$ higher than that for low-risk CKD. Emergency visit mean annual costs were $\$ 14$ (1.1 times; $95 \%$ CI $\$ 22-49 ; P=0.455), \$ 131$ (2.2 times; 95\% CI $\$ 76-185 ; P<0.001)$ and $\$ 243$ (3.2 times; 95\% CI $\$ 175-311 ; P<0.001)$ higher for moderate, high, and very high risk, respectively when compared to low-risk CKD.

\section{Relationships between T2DM CKD and mean annualdirect medical costs}

CKD, age at study entry, Malay ethnicity, duration of DM, use of statins/ fibrates, and modified DCSI were found to be positively associated with an increase in mean annual direct medical costs in the univariate analysis. The associations persisted for CKD and modified DCSI in the fully adjusted model. In addition, use of oral medication only, as well as both oral medication and insulin, were surprisingly negatively associated with mean annual total costs in Table 5. There was no significant association between gender and mean annual total costs in the unadjusted analysis. The association between ethnicity groups, DM duration, use of RAS antagonist and use of statins/ fibrates with mean annual total cost was attenuated and lost statistical significance in the fully adjusted model (Tables 5 and 6).

Table 4 showed that patients with CKD had 2.2 times higher total mean annual costs than patients without CKD (exponentiated coefficient $(\exp (\beta)) 2.22(95 \%$ CI 1.87-2.65); $P<0.001)$. CKD was associated with 1.7 times higher total mean annual costs than non-CKD $(\exp (\beta) 1.65(95 \%$ CI $1.34-2.03 ; P<0.001)$ in the fully adjusted model. Compared to the low-risk group, moderate $(P=0.003)$, high-risk $(P<0.001)$ and very high-risk $(P<0.001)$ groups of CKD were positively associated with mean annual total costs in the univariate analysis. Compared to low-risk CKD, moderate risk,

Table 5. Association between CKD and mean annual total costs $(\mathrm{n}=1275)$

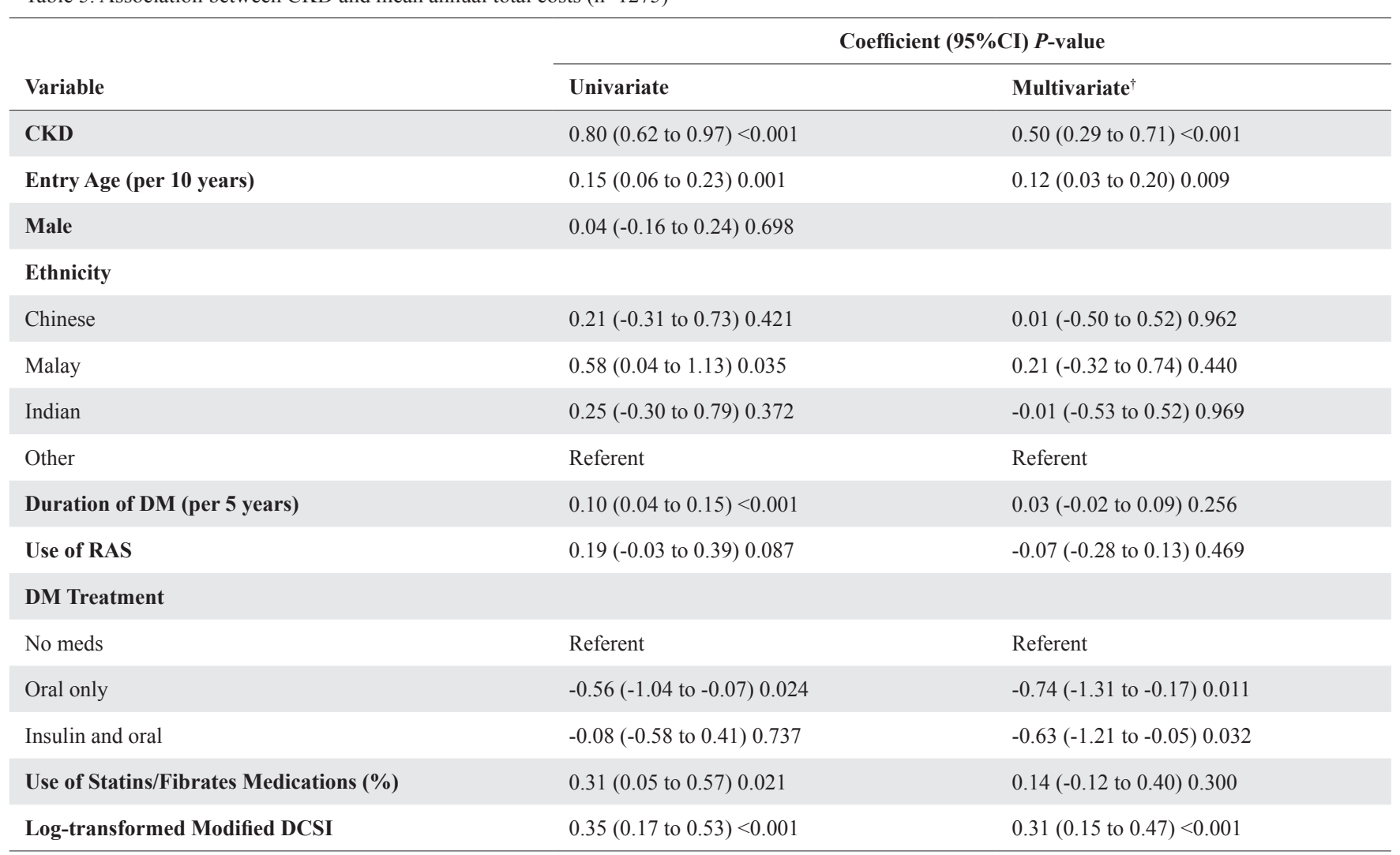

CKD: chronic kidney disease; DCSI: diabetes complications severity index; DM: diabetes mellitus; RAS: renin-angiotensin system antagonist

${ }^{\dagger}$ Adjusted for age, ethnicity, duration of DM, use of RAS antagonist, DM treatment, use of statins/fibrates and log-transformed modified DCSI score 
Table 6. Association between CKD severity and mean annual total costs $(n=1275)$

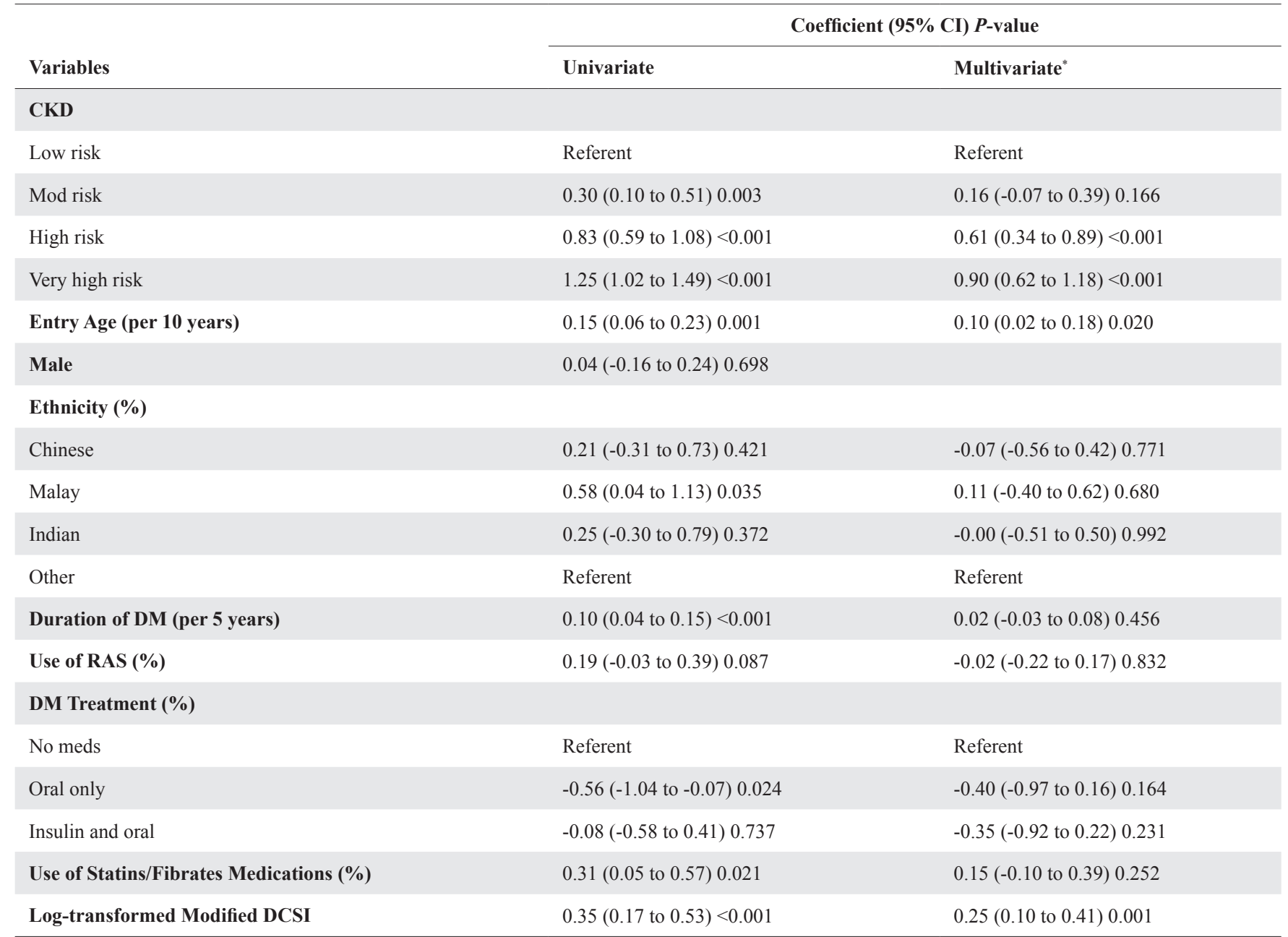

CKD: chronic kidney disease; DCSI: diabetes complications severity index; DM: diabetes mellitus: RAS, renin-angiotensin system antagonist

"Adjusted for age, ethnicity, duration of DM, use of RAS antagonist, DM treatment, use of statins/fibrates and log-transformed modified DCSI score.

high-risk and very high-risk CKD were associated with 1.4 times $[\exp (\beta) 1.36(95 \%$ CI $1.11-1.66 ; P=0.003)]$, 2.3 times $[\exp (\beta) 2.30(95 \%$ CI $1.80-2.95 ; P<0.001)]$ and 3.5 times $[\exp (\beta) 3.50(95 \%$ CI $2.76-4.34 ; P<0.001)]$ higher mean annual total cost respectively. The association persisted for high and very high-risk CKD in the fully adjusted model $(P<0.001)$ (Table 6). Patients with high-risk and very high-risk CKD had 1.8 times $[\exp (\beta) 1.84(95 \%$ CI 1.40-2.42; $P<0.001]$ and 2.5 times $[\exp (\beta) 2.46(95 \%$ CI 1.86-3.26; $P<0.001]$ higher mean annual total cost than those with low-risk CKD.

\section{Discussion}

In this study of a multi-ethnic population in Singapore, CKD occurred in $57.3 \%$ of patients of which patients with CKD had total higher median medical cost than those without $\mathrm{CKD}(\$ 1,571$, interquartile range of $\$ 885-3,411$ vs $\$ 949$ (\$461-1,669); $P<0.001)$. We found that the presence and increased severity of CKD in T2DM patients were independently associated with an increase in direct medical costs, in spite of correcting for DCSI, indicating that more resources were utilised by patients with $\mathrm{CKD}$. These results are aligned with previous cross-sectional studies, which demonstrated that medical costs rose with increased severity of CKD. Laliberté reported that the total direct all-cause healthcare costs were significantly higher for T2DM patients with CKD at US\$11,814 (ratio of CKD/non-CKD 2.8 times) and US\$10,625 (ratio of CKD/non-CKD 2.0 times) for T2DM patients 
with both CKD and hypertension. ${ }^{9}$ Furthermore, Vupputuri et al. also reported that the corresponding total baseline annual costs for CKD stage 0-2, 3 and 4 were US $\$ 8,206$, US $\$ 12,529$ and US $\$ 23,229$, respectively for each patient. ${ }^{12}$ This works out to be 1.5 times higher for CKD stage 3 and 2.8 times higher for CKD stage 4 compared to CKD stage $0-2$. Our results are in line with these findings. ${ }^{12}$ The stepwise increase in direct medical costs with worsening of CKD categories highlights the importance of screening for DM and treatment for retardation of the disease progression as they are potentially cost saving..$^{27,28}$

In our study, outpatient costs for low- and moderaterisk groups were higher than that of their inpatient costs. As explained by Goncalves et al., CKD is largely treated in the outpatient setting in Brazil. ${ }^{16}$

In contrast, inpatient costs for high-risk and very high-risk groups of patients were the major drivers of cost. Low et al. also reported that patients with CKD of increased severity have a higher propensity of having decompensation of their condition, thereby being more likely to incur increased healthcare expenditure to treat their CKD-related conditions. ${ }^{10}$ As such, inpatient costs are the major type of resources consumed. Satyavani et al. reported that T2DM patients with CKD prior to ESRD incurred higher costs on hospital admissions compared to T2DM patients without complications in India. ${ }^{11}$ Similarly, Laliberté also corroborated that hospitalisations contribute most to the healthcare cost differences between CKD and non-CKD groups. ${ }^{9}$ In the study by Jiang et al., higher inpatient admission costs and outpatient costs drove the increase in DM-related healthcare costs among patients with increasing comorbidity. ${ }^{20}$ In particular, patients who reached endstage renal disease have a substantially increased chance of attendance in the emergency department with subsequent hospitalisation due to acute complications and urgent haemodialysis, resulting in a more than 5 -fold increase in medical costs during the first year of dialysis. ${ }^{29}$ Moreover, higher mortality among diabetic patients with higher CKD risk categories also contributes to higher inpatient cost. ${ }^{30}$

The age at study entry and duration of DM (in unadjusted analysis) were significant in incurring increased hospital expenditure for T2DM patients with CKD. Longer duration of DM may have been associated with an increase in DM complications such as more severe CKD, thereby incurring higher costs. Gonclaves et al. suggested that the age of 65-75 years was an important factor that contributed to the development of Diabetes-related end-stage kidney disease (ESKD). ${ }^{16}$ The increased prevalence of DM and relative risk of developing ESKD was found to be present in the elderly diabetic Brazilian population.

SBP, HbAlc and insulin usage were also associated with higher severity of CKD. This is supported by Tan et al., where a history of hypertension, and a higher $\mathrm{HbAlc}$ baseline were found to be significant and independent risk factors associated with progression to albuminuria in DM patients. ${ }^{31}$ A study by Low et al. showed that only $30.9 \%$ of patients in Singapore met the target $\mathrm{HbA} 1 \mathrm{c}<7 \%$, and $53.4 \%$ had $\mathrm{BP}<140 / 80$ mmHg. ${ }^{32}$ While current clinical practice guidelines have evolved to recommend less intensive glycaemic control in DM patients with more comorbidities (CKD and cardiovascular) to avoid the paradoxical increase in mortality possibly attributed to severe hypoglycaemia, it would still be useful to keep glycaemia and blood pressure under control.

Interestingly, DM medications (oral only, and oral plus insulin) were associated with lower mean annual total costs. Liu et al. reinforced the importance of glycaemic control in slowing down diabetes progression (in patients with low risk of hypoglycaemia) by reducing glucotoxicity, whereby resolving hyperglycaemia in itself might improve insulin secretion. ${ }^{33}$ Better glycaemic control may retard further CKD progression, which may also lead to lower direct medical costs over time. As these factors are potentially modifiable, it is pertinent to highlight their importance during patient education and clinical management. Conversely, another possible explanation for the lower mean annual total costs would be the paradoxically reduced reliance on anti-diabetic medications to achieve glycaemic control target with advancing CKD, especially in stages 4 and 5 before the initiation of renal replacement therapy, which was associated with high-cost utilisation from treatment needs other than glycaemic control. In contrast, those who require multiple anti-diabetic medications tend to have milder CKD and thus a reduced overall cost.

To our knowledge, this is the first study that examines the relationship between the presence and severity of diabetes-induced CKD with direct medical costs in Singapore, with the one other study in Singapore conducted by Low et al. examining the relationship between the progression of diabetic kidney disease with direct medical costs. This study provides information on actual direct medical costs incurred by each patient, and helps to inform cost-effectiveness analysis of 
interventions to delay the progression of CKD. Laboratory results were also available for analysis to enable us to ascertain the CKD status and risk.

However, there is a lack of information on indirect costs (i.e. transport) and intangible costs (i.e. productivity losses associated with absenteeism, presenteeism and premature mortality). Furthermore, the observational nature of the study precludes us from asserting a causal association between CKD and higher medical costs. Finally, our study was also based on patients with T2DM in an acute care hospital, whose conditions may vary from the Singaporean population of diabetic patients at a national level. Our findings thus cannot be generalised to other settings such as primary care polyclinics and general practitioners (GPs). There has also been an evolving trend to manage chronic disease patients in primary care to ease the congestion at specialist outpatient clinics. ${ }^{34}$ George et al. explained in his study that the majority of primary healthcare physicians reported screening for CKD. ${ }^{35}$ However, only $38 \%$ of them were aware of or adhering to CKD guidelines. This suggests that the GPs who were unaware of CKD clinical guidelines are less likely be able to recognise CKD progression and recommend nephrologist care. Information such as the cost of right-siting CKD care, and costs of transferring care are unavailable. Lastly, we lack information on patient survival. Therefore we are unable to estimate the lifetime additional direct medical costs.

In conclusion, the presence and increased severity of CKD is significantly associated with higher direct medical costs in T2DM patients. Actively preventing the occurrence and progression of DM-induced CKD may significantly reduce the consumption of healthcare resources and healthcare costs. Even though maintaining good control of CKD results in an increased usage of healthcare services, this can lead to savings in healthcare expenditure in the long run. Thus, intensive efforts to treat and slow down the progression of CKD may be crucial to reducing medical costs.

\section{REFERENCES}

1. Root HF, Story RD, Cortesi JB. Diabetic coma versus diabetic nephropathy; hazards of factitious insulin resistance, oliguria and hyperkalemia. N Engl J Med 1951;245:765-70.

2. Phan TP, Alkema L, Tai ES, et al. Forecasting the burden of type 2 diabetes in Singapore using a demographic epidemiological model of Singapore. BMJ Open Diabetes Res Care 2014;2:e000012.

3. Png ME, Yoong J, Phan TP, et al. Current and future economic burden of diabetes among working-age adults in Asia: conservative estimates for Singapore from 2010-2050. BMC Public Health 2016;16:153.
4. Brown JB, Pedula KL, Bakst AW. The progressive cost of complications in type 2 diabetes mellitus. Arch Intern Med 1999; 159:1873-80.

5. Zhuo X, Zhang P, Hoerger TJ. Lifetime direct medical costs of treating type 2 diabetes and diabetic complications. Am J Prev Med 2013;45:253-61.

6. Morsanutto A, Berto P, Lopatriello S, et al. Major complications have an impact on total annual medical cost of diabetes: results of a database analysis. J Diabetes Complications 2006;20:163-9.

7. Pena MJ, de Zeeuw D, Mischak H, et al. Prognostic clinical and molecular biomarkers of renal disease in type 2 diabetes. Nephrol Dial Transplant 2015;30 Suppl 4:iv86-95.

8. United States Renal Data System. Chapter 11: International Comparisons 2017. Available at: https:/www.usrds.org/media/1738/ v2_c11_intcomp_18_usrds.pdf

9. Laliberte F, Bookhart BK, Vekeman F, et al. Direct all-cause health care costs associated with chronic kidney disease in patients with diabetes and hypertension: a managed care perspective. J Manag Care Pharm 2009;15:312-22.

10. Low S, Su Chi L, Zhang X, et al. Medical Costs Associated with Chronic Kidney Disease Progression in an Asian Population with Type 2 Diabetes Mellitus. Nephrology (Carlton) 2019;24:534-41.

11. Satyavani K, Kothandan H, Jayaraman M, et al. Direct costs associated with chronic kidney disease among type 2 diabetic patients in India. Indian J Nephrol 2014;24:141-7.

12. Vupputuri S, Kimes TM, Calloway MO, et al. The economic burden of progressive chronic kidney disease among patients with type 2 diabetes. J Diabetes Complications 2014;28:10-6.

13. Azmi S, Goh A, Muhammad NA, et al. The Cost and Quality of Life of Malaysian Type 2 Diabetes Mellitus Patients with Chronic Kidney Disease and Anemia. Value Health Reg Issues 2018;15:42-9.

14. Nichols GA, Vupputuri S, Lau H. Medical care costs associated with progression of diabetic nephropathy. Diabetes Care 2011; 34:2374-8.

15. Ozieh MN, Dismuke CE, Lynch CP, et al. Medical care e xpenditures associated with chronic kidney disease in adults with diabetes: United States 2011. Diabetes Res Clin Pract 2015; 109:185-90.

16. Goncalves GMR, da Silva EN. Cost of chronic kidney disease attributable to diabetes from the perspective of the Brazilian Unified Health System. PloS One 2018;13:e0203992.

17. Levin A, Stevens PE, Bilous RW, et al. Kidney disease: Improving global outcomes (KDIGO) CKD work group. KDIGO 2012 clinical practice guideline for the evaluation and management of chronic kidney disease. Kidney Int Suppl 2013;3:1-150. https://doi. org/10.1038/kisup.2012.73

18. Stevens PE, Levin A. Evaluation and management of chronic kidney disease: synopsis of the kidney disease: improving global outcomes 2012 clinical practice guideline. Ann Intern Med 2013;158:825-30.

19. Young BA, Lin E, Von Korff M, et al. Diabetes complications severity index and risk of mortality, hospitalization, and healthcare utilization. Am J Manag Care 2008;14:15-23.

20. Jiang R, Law E, Zhou Z, et al. Clinical Trajectories, Healthcare Resource Use, and Costs of Diabetic Nephropathy Among Patients with Type 2 Diabetes: A Latent Class Analysis. Diabetes Ther 2018;9:1021-36.

21. Liu JJ, Tavintharan S, Yeoh LY, et al. High normal albuminuria is independently associated with aortic stiffness in patients with Type 2 diabetes. Diabet Med 2014;31:1199-204. 
22. Pek SL, Tavintharan S, Wang X, et al. Elevation of a novel angiogenic factor, leucine-rich-alpha2-glycoprotein (LRG1), is associated with arterial stiffness, endothelial dysfunction, and peripheral arterial disease in patients with type 2 diabetes. J Clin Endocrinol Metab 2015;100:1586-93.

23. Tavintharan S, Ning $\mathrm{C}, \mathrm{Su} \mathrm{Chi}$ L, et al. Prevalence and risk factors for peripheral artery disease in an Asian population with diabetes mellitus. Diab Vasc Dis Res 2009;6:80-6.

24. Aboyans V, Criqui MH, Abraham P, et al. Measurement and interpretation of the ankle-brachial index: a scientific statement from the American Heart Association. Circulation 2012;126:2890-909.

25. Wu CX, Tan WS, Toh MP, et al. Stratifying healthcare costs using the Diabetes Complication Severity Index. J Diabetes Complications 2012;26:107-12.

26. Glasheen WP, Renda A, Dong Y. Diabetes Complications Severity Index (DCSI)-Update and ICD-10 translation. J Diabetes Complications 2017;31:1007-13.

27. Wu B, Zhang S, Lin $\mathrm{H}$, et al. Prevention of renal failure in Chinese patients with newly diagnosed type 2 diabetes: A costeffectiveness analysis. J Diabetes Investig 2018;9:152-61.

28. Golan L, Birkmeyer JD, Welch HG. The cost-effectiveness of treating all patients with type 2 diabetes with angiotensin-converting enzyme inhibitors. Ann Intern Med 1999;131:660-7.
29. Jiao F, Wong CKH, Tang SCW, et al. Annual direct medical costs associated with diabetes-related complications in the event year and in subsequent years in Hong Kong. Diabet Med 2017; 34:1276-83.

30. Wen CP, Cheng TY, Tsai MK, et al. All-cause mortality attributable to chronic kidney disease: a prospective cohort study based on 462293 adults in Taiwan. Lancet 2008;371:2173-82.

31. Tan S, Wong LY, Toh MP. Incipient Albuminuria in Persons with Newly Diagnosed Type 2 Diabetes Mellitus: A 5-Year Retrospective Cohort Study. Ann Acad Med Singap 2018;47:502-8.

32. Low SK, Sum CF, Yeoh LY, et al. Prevalence of Chronic Kidney Disease in Adults with Type 2 Diabetes Mellitus Ann Acad Med Singap 2015;44:164-71.

33. Liu S, Liu JJ, Gurung RL, et al. Clinical Determinants of Diabetes Progression in Multiethnic Asians with Type 2 Diabetes-A 3-Year Prospective Cohort Study. Ann Acad Med Singap 2019; 48:217-23.

34. Wee SL, Tan CG, Ng HS, et al. Diabetes outcomes in specialist and general practitioner settings in Singapore: challenges of right-siting. Ann Acad Med Singap 2008;37:929.

35. George PP, Oh CM, Loh PT, et al. Right-siting chronic kidney disease care-a survey of general practitioners in Singapore. Ann Acad Med Singap 2013;42:646-56. 